\title{
Predicting treatment course and outcome using a promotion and prevention framework in a community sample of arthritis sufferers
}

This article was published in the following Dove Press journal:

Patient Preference and Adherence

\author{
Dan V Blalock ${ }^{1,2}$ \\ Patrick E McKnight ${ }^{3}$ \\ Todd B Kashdan ${ }^{3}$ \\ Simone C Franz ${ }^{3,4}$ \\ 'Center for Health Services Research \\ in Primary Care, Durham Veterans \\ Affairs Medical Center, Durham, NC, \\ USA; ${ }^{2}$ Department of Psychiatry and \\ Behavioral Sciences, Duke University \\ School of Medicine, Durham, NC, \\ USA; ${ }^{3}$ Department of Psychology, \\ George Mason University, Fairfax, VA, \\ USA; ${ }^{4}$ Humanproof, LLC, Arlington, \\ VA, USA
}

Objective: The present study examined the proposition that patients need to focus on something beyond simply "getting better". In a sample of arthritis sufferers, we distinguished individuals by the goals that motivated them - moving toward aspirational goals and maximizing gains (promotion focus) rather than obligations and minimizing losses (prevention focus) - and how these motivational styles influenced treatment.

Methods: Patients $(\mathrm{N}=254)$ participated in a randomized controlled trial of resistance training and self-management, providing 6 time points of data over 2 years. Promotion and prevention focus at baseline were used to predict the course (compliance and changes in coping self-efficacy) and outcome (changes in physical functioning) of treatment.

Results: Arthritis sufferers with strong promotion orientations showed significant improvements in physical functioning (a direct positive impact on physical health); there were no significant associations with treatment compliance and coping self-efficacy. Arthritis sufferers with strong prevention orientations complied less with the treatment and showed little change in coping self-efficacy during treatment, which, in turn, predicted worse physical functioning over time (a pernicious, indirect influence on treatment outcome).

Conclusion: A focus on positive approach-oriented goals may improve overall treatment response, whereas a focus on negative avoidance-oriented goals may degrade treatment response through reduced compliance and self-efficacy.

Keywords: treatment, promotion and prevention, self-efficacy, physical functioning, arthritis

\section{Introduction}

Consider two patients seeking medical advice for knee pain: patient A seeks treatment to "get better" by preventing distress caused by her symptoms, while patient B seeks treatment to "get better" by promoting a more active and meaningful lifestyle. Both patients received a prescription pain reliever, yet patient $\mathrm{B}$ remains unhappy and incapable of striving toward fulfillment. Her initial treatment response - preventing distress - did not lead her to her real desired outcome - promoting an active lifestyle. Most clinical psychology theories rest on the assumption that the distress reduction leads to desirable, positive life outcomes. Yet in this example, the patient's initial goal orientation when seeking treatment (preventing a negative outcome versus promoting a positive outcome) was a driving factor in the process and outcome of treatment. Thus, reduction in pain does not necessarily lead to an increase in positive experiences.

Indeed, researchers in various fields have discovered that the absence of negative outcomes, events, thoughts, and emotions does not necessarily imply the presence of positive outcomes, events, thoughts, and emotions. ${ }^{1,2}$ Much work has illustrated the
Correspondence: Dan V Blalock Center for Health Services Research and Development, Durham Veterans Affairs Medical Center, 4II West Chapel Hill Street, Suite 600, Durham,

NC 2770I, USA

Tel + I 919286041 I

$\mathrm{Fax}+19194165836$

Email dvb@duke.edu 
independence of positive and negative motivation systems (eg, behavioral activation/inhibition) as well as the independence of motivational states initiated by positive and negative goals - namely promotion and prevention orientations. ${ }^{3}$ These different motivational systems produced different behavioral outcomes and lead to novel treatments for physical and psychological health. ${ }^{4}$

Despite these results, no studies have examined how these motivational systems work for patients whose treatment typically involves the prevention goal of reducing pain - patients with osteoarthritis. The closest approximations have examined how depression (which has been argued as an underdeveloped promotion orientation $)^{5}$ is associated with poorer osteoarthritis treatment outcomes, ${ }^{6}$ how selfdiscrepancies between current and idealized selves predict more pain in osteoarthritis, ${ }^{7}$ and how positive and negative beliefs about treatment differentially influence those processes in osteoarthritis patients. ${ }^{8}$

The purpose of this paper was to examine these independent goals and orientations to determine if (and how) individuals gain more from treatment when the treatment matches the patient's motivational orientation. A promotion and prevention orientation is a potentially informative framework because prior work showed differential outcomes based upon an individual's goals, as well as processes and strategies used to reach those goals. ${ }^{9-11}$ Unfortunately, the patient burden of such self-report instruments often preclude patient-reported outcomes (PRO's) such as these from being assessed outside of certain explicit PRO initiatives, ${ }^{12}$ and so alternative tools such as the nomogram approach used in the present research can help examine these broad orientations without increasing patient burden. ${ }^{13}$

\section{Treatment matching with promotion/ prevention orientations}

Many researchers posit that when treatments match a patient's preferences or aptitudes (cf. aptitude by treatment interactions), ${ }^{14}$ the treatment effects get maximized. Those expectations, however, have not had much empirical support over the years. Individual difference variables often reduce the effects of treatments and few findings suggest that there are positive factors that amplify treatment effects. Promotion and prevention orientations stand as one example that defies those non-findings (Regulatory Fit). ${ }^{15}$ A promotion-oriented person eagerly seeks out gains by focusing on achievement and growth-related goals. By contrast, a prevention-oriented person attempts to avoid losses by focusing on responsibility and safety-related goals. ${ }^{9}$ When viewed in this light, the absence of a prevention goal clearly does not imply the presence of a promotion goal and vice versa. Yet when a person navigates life by heavily leaning on one of these orientations, this individual difference may offer researchers (and practitioners) a simple heuristic for matching treatments to patients to maximize treatment outcomes.

Each of these factors influencing treatment requires information that is 1) hard to gather from patients and 2) difficult to apply to individuals. A patient's promotion/prevention orientation, however, remains relatively easy to measure and use for classifying patients. In addition, promotion and prevention orientations likely contribute in unique ways to the treatment process and therefore to treatment outcomes; so, we examined the mechanisms by which a person's promotion/prevention orientation influences treatment outcomes. By examining both outcome and process, this research provides inroads for better matching patients with a broader range of tailored treatments.

\section{Clinical application of promotion/ prevention - self-efficacy}

A primary focus in many treatments is increasing a patient's self-efficacy regarding their ability to make improvements. A person's belief in their ability to achieve some outcome (ie, self-efficacy) is crucial to the change process. Selfefficacy leads to improvement in a variety of domains, from increasing well-being, to fostering resilience, to facilitating recovery. ${ }^{16}$ The treatment process, however, involves reciprocal relationships between objective gains and perceived self-efficacy. To this end, increases in self-efficacy can be seen as crucial to increases in functional outcomes - a relationship that has been established through experimental manipulations. ${ }^{17}$ Given that individuals in treatment may have differing goals (promotion or prevention goals), they likely have differing levels of self-efficacy and goal-related treatment trajectories throughout the course of treatment. Differing levels of self-efficacy throughout the treatment process potentially influence behavioral shifts in response to treatment. These behavioral shifts may influence changes in functional outcomes without being directly responsible for functional change.

\section{Clinical application of promotion/ prevention - compliance}

Aside from the cognitive element of self-efficacy, another important treatment mechanism is the behavioral component of compliance. Research has demonstrated a strong connection between types of health messages and individuals' 
compliance with these messages. Health messages framed in terms of gains (promotion) or losses (prevention) can lead people to comply in different ways. ${ }^{18}$ Gain-framed health messages lead people to more health-promotion behaviors; loss-framed health messages lead people to more diseaseprevention behaviors. These broad message manipulations, however, may only have a small effect, ${ }^{19}$ and it is often the case that only one particular type of health behavior is relevant to a person, such as altering alcohol intake, instead of preventing a disease, such as cancer. The greatest benefit might arise from targeting specific health behaviors with attention to individual differences in motivational dispositions. Recent research suggests that tailoring health messages to individuals' motivational dispositions increases positive attitudes toward the health behaviors,${ }^{20}$ intention to perform the health behaviors, ${ }^{21}$ and actual compliance with the healthy behaviors. $^{22}$ Our goal in the present research study was to assess the usefulness of promotion and prevention dispositions in the treatment of arthritis.

\section{Present study}

Interventions aimed at treating a physically debilitating, degenerative disease such as arthritis serve as a useful context where individual differences (promotion or prevention) might impact processes (eg, compliance, expectations) and outcomes (eg, immediate treatment response, long-term impact). One person may seek treatment with the goal of preventing the pain associated with arthritis (much like patient A), another person will seek treatment with the goal of promoting their active lifestyle that has been hindered by the pain of arthritis (much like patient B). Treatments framed to favor only one of these orientations ought to produce greater treatment response variability. ${ }^{23,24}$ Many treatment outcomes for arthritis are overt and readily measurable (eg, physical movement/functioning). Thus, arthritis treatment offers us a unique opportunity to test our primary proposition: promotion and prevention orientations both predict treatment response but in unique ways.

The present study explores the independent contributions of promotion and prevention goal orientations to predict how people with osteoarthritis comply with, feel competent about, and respond to treatment. Our outcome variables - total treatment compliance, changes in selfefficacy, and changes in physical functioning - reflect greater engagement, and so we expected promotion orientation to be a strong positive predictor for all three. By contrast, we expected prevention orientation to negatively predict all three outcomes. Furthermore, our compliance ratings reflect a trait-dependent outcome, whereas changes over time in self-efficacy and physical functioning measures reflect statedependent outcomes. As a result, we hypothesized that people with a stronger promotion orientation will show higher overall treatment compliance and greater positive changes in coping self-efficacy leading to greater positive changes in physical functioning (via a mediation model); a stronger prevention orientation was expected to be a negative predictor of overall compliance, changes in self-efficacy, and changes in functioning. In sum, we aimed to identify distinct connections between a fundamental individual difference factor (promotion/prevention) and common treatment processes (compliance, self-efficacy, and physical functioning), so that treatment of arthritis as a disease can be refined to a more targeted treatment of individuals with arthritis.

More precisely, we hypothesized a mediation model where a promotion and prevention orientation would predict treatment compliance as well as changes in coping selfefficacy and functional outcomes. The treatment outcome effects were expected to be mediated by treatment compliance and changes in coping self-efficacy. Hence, our model specifies a simplex mediation process whereby both treatment compliance and changes in coping self-efficacy mediate the effects of a promotion orientation. We included both promotion and prevention orientation in the model at the same level and allowed them to correlate. Testing both orientations simultaneously provides a more conservative test for the relative influence of both promotion and prevention orientation, without biasing the model toward one or the other.

\section{Methods}

We tested the aforementioned mediation model with a sample of patients undergoing arthritis treatment. Our sample represented both a common medical problem as well as treatment outcomes that can be characterized as subjective and objective. We provide further justification for the utility of this sample in the following text.

Osteoarthritis, the most common form of arthritis, currently affects about $12 \%$ of the US population. ${ }^{25}$ Several efficacious treatments exist that reliably provide relief to sufferers (eg, exercise ${ }^{26,27}$ self-management; ${ }^{28}$ anti-inflammatory drugs), ${ }^{29}$ but treatment effect sizes (between 0.10 and 0.40 ) indicate that a sizable percentage of osteoarthritis patients fail to respond to these treatments; conservative estimates of number needed to treat lead us to expect $50 \%-90 \%$ treatment non-response. Considering individual differences that enhance treatment outcomes might produce a large number (ie, millions) of previously unrealized positive 
treatment outcomes. Furthermore, if this individual difference framework can aid in treatment with such low response rates, it may excel in aiding treatments with much higher response rates (ie, cognitive and behavioral therapies).

\section{Data and design}

Two-hundred and fifty-four participants $(\mathrm{N}=254)$ provided 2-year repeated measures (6 time points) data from a nonblinded, randomized controlled trial of resistance training and self-management for osteoarthritis (the Knee Study; 273 patients were originally randomized, see Figure 1); these data served as the basis for our current study. The original study was approved by the University of Arizona Institutional Review Board and conducted in compliance with the Helsinki Declaration at the University of Arizona Arthritis Center in Tucson, AZ, USA. All study participants gave written informed consent prior to randomization. The trial aimed to compare the effects of three interventions: a resistance training program, a self-management program, and a combined resistance training and self-management program over a 24 -month period. ${ }^{30}$ All three treatment arms were designed to improve coping self-efficacy and physical functioning. We found no significant differences between treatment arms across time on the relevant variables for this study, and all treatment arms showed significant changes over time. Accordingly, all current data analyses ignore between treatment arm effects, but include the change over time.

\section{Participants}

Knee Study participant eligibility criteria were 1) between the age of 35 and 64 years; 2) reported pain on most days in 1 or both knees; 3 ) duration of symptoms of $<5$ years; 4) had Kellgren and Lawrence classification (KL) grade II radiographic evidence of knee osteoarthritis in one or both knees; ${ }^{31}$ and 5) self-reported disability due to knee pain for at least 3 of the following: descending or ascending stairs, walking, kneeling, or performing daily activities. Potential participants were excluded if they had 1) an uncontrolled medical condition that precluded safe participation or prevented completion of the study (eg, heart disease, blood pressure, or respiratory conditions); 2) any neurological condition that could affect coordination; 3) inflammatory arthritis (eg, rheumatoid or psoriatic arthritis); 4) previous knee surgery; 5) KL grades III or IV radiographic evidence of osteoarthritis in one or both knees; 6) body mass index (BMI) $>37.5 \mathrm{~kg} / \mathrm{m}^{2}$ - individuals over that limit were advised to follow a weight loss program and achieve stable weight for 6 months prior to participation; 7) a knee corticosteroid injection in the previous 3 months; 8) plans to move from the local area; 9) plans to become pregnant during the study period; 10) more than 120 minutes per week of any vigorous (eg, exercise, walking, household chores, etc.) physical activity; or 11) participated in any form of resistance training. Staff recruited participants from the local community, assessed eligibility by telephone, and if appropriate scheduled them for a radiographic exam administered by a staff rheumatologist. Individuals meeting all eligibility criteria were followed for a run-in period (average run-in days=73) and then randomly assigned to one of three treatment groups - a resistance training group, a self-management group, or a combined treatment group. Males and females were allocated separately in random blocks to ensure an equivalent distribution of sex among the groups.

\section{Measures}

\section{Promotion orientation}

We combined 20 total items from three scales to serve as our composite promotion orientation (Table 1). Each item was coded to correspond with a specific item from the Regulatory Focus Questionnaire promotion scale (RFQ). ${ }^{32}$ For example, the Center for Epidemiologic Studies Depression Scale (CES-D) item eight ("I felt hopeful about the future") was matched with RFQ item 10 ("I feel like I have made progress toward being successful in my life"). This composite included six items from the Revised NEO Personality Inventory (NEO-PI-R) short form (baseline administration only), ${ }^{33}$ all 10 items from the positive affect subscale of the Positive and Negative Affect Scale (PANAS; ${ }^{34}$ administered four times over 2 years), and four items from CES-D (administered four times over 2 years), ${ }^{35}$ all of which were positively worded and positively scored, regardless of the scales' reverse scoring. All items from each of these three scales corresponded with specific items on the promotion subscale of the RFQ. Each variable was standardized and then combined by taking the mean standardized variable to form a unit-weighted score $\left(\sigma^{2}=0.37\right) .{ }^{36}$ The internal consistency of the promotion-relevant measures was high $(\alpha=0.90)$ indicating good reliability and within the range of many other composite measures. Additionally, we assessed whether the newly created promotion scale changed over time but time failed to predict any differences in a linear regression and, as a result, we pooled all repeated measures into a within-subject mean.

\section{Prevention orientation}

We combined 18 total different items from the three scales mentioned previously for promotion and these items served as our composite prevention orientation measure (Table 1). 


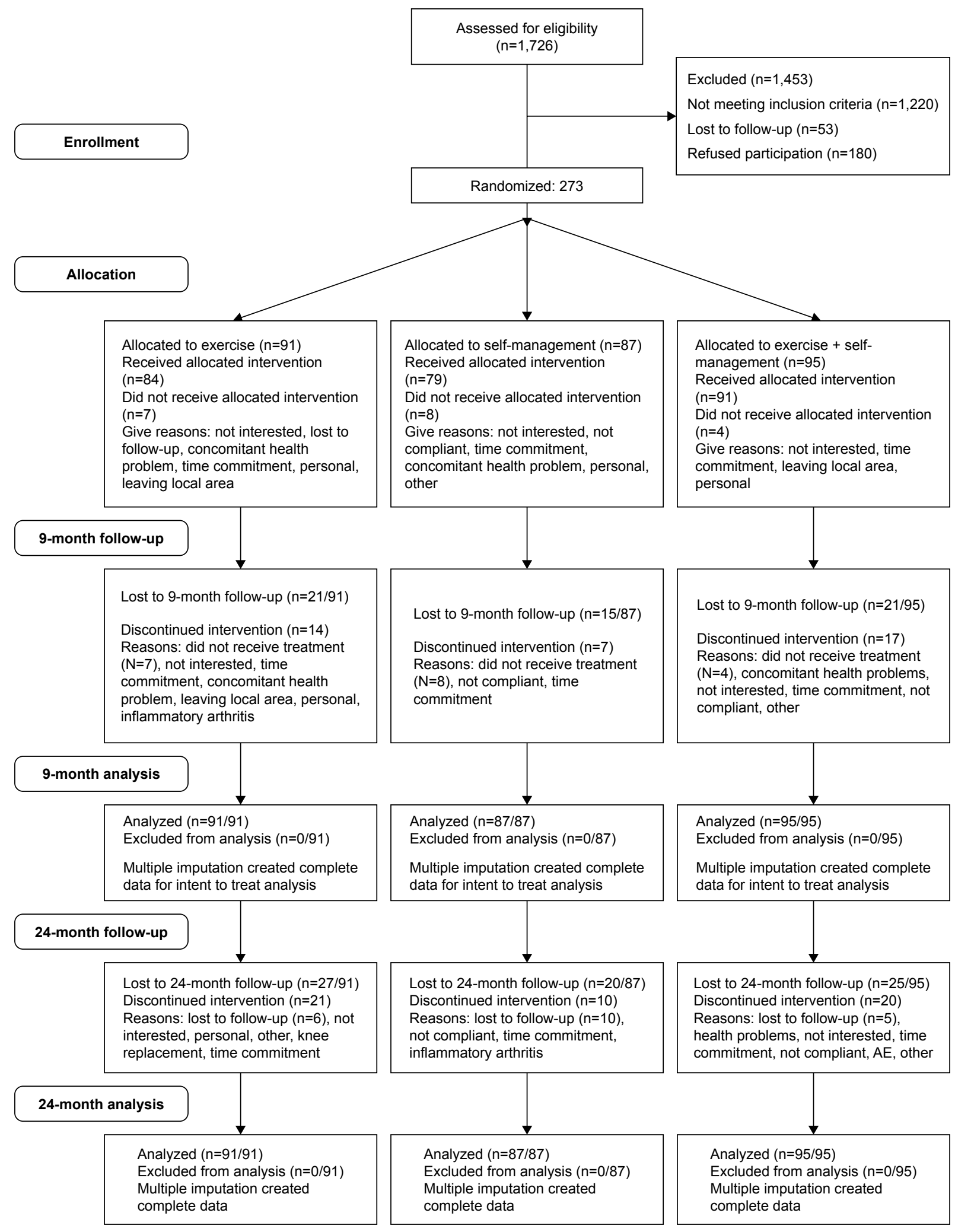

Figure I Consort diagram.

Each item was coded to correspond with a specific item from the RFQ prevention scale. For example, Neo-Pi-R item 10 ("I often feel tense and jittery") was matched with RFQ item five ("How often do you obey rules and regulations that were established by your parents?"). The composite included five items from the NEO-PI-R short form, all 10 items from the negative affect subscale of PANAS, and three items from the CES-D. Likewise, we used the same unit-weighted factor 
Table I Item maps for composite measures of promotion and prevention

\begin{tabular}{lllll}
\hline $\begin{array}{l}\text { RFQ } \\
\text { subscale }\end{array}$ & $\begin{array}{l}\text { RFQ } \\
\text { item }\end{array}$ & $\begin{array}{l}\text { CES-D } \\
\text { item }\end{array}$ & $\begin{array}{l}\text { Neo-Pi-R } \\
\text { item }\end{array}$ & $\begin{array}{l}\text { PANAS } \\
\text { item }\end{array}$ \\
\hline Promotion & I(R) & 4,16 & 3 & 5,16 \\
Promotion & 3 & - & 16 & $3,9,12$ \\
Promotion & 7 & - & - & 14 \\
Promotion & $9(\mathrm{R})$ & - & - & 17 \\
Promotion & I0 & 8 & - & 10 \\
Promotion & II (R) & 12 & $1,6,19,33$ & 1,19 \\
Prevention & $2(\mathrm{R})$ & 10 & 21,22 & 8,20 \\
Prevention & $4(\mathrm{R})$ & - & 18 & 2,6 \\
Prevention & 5 & - & 10 & 7,18 \\
Prevention & $6(\mathrm{R})$ & - & - & 13 \\
Prevention & $8(\mathrm{R})$ & $5,1 \mathrm{I}$ & 27 & $4,1 \mathrm{I}, 15$ \\
\hline
\end{tabular}

Notes: All composite items were positively scored. “-” = not applicable.

Abbreviations: RFQ, Regulatory Focus Questionnaire promotion scale; CES-D, Center for Epidemiologic Studies Depression Scale; Neo-Pi-R, Revised NEO Personality Inventory; PANAS, Positive and Negative Affect Scale; R, reverse-coded RFQ items.

scoring method as discussed for the promotion orientation scale $\left(\sigma^{2}=0.32\right)$. All items from each of these four scales were positively worded and positively scored, regardless of the scales' reverse scoring, and corresponded with specific items on the prevention subscale of the RFQ. The internal consistency of these four measures was reasonably high $(\alpha=0.84)$ indicating high intercorrelations among the variables. Similar to the promotion orientation measure, we assessed the temporal stability of the scores by person over time and found no significant changes; thus, we combined the repeated measures into a within-subject mean.

\section{Mediators}

Two constructs - overall treatment compliance and coping self-efficacy changes - provided the specific mechanistic variables for our mediational model. Treatment staff recorded treatment compliance by both attendance and successful completion (ie, participation and attendance) of every treatment occasion and scored compliance on a 0-100 scale with 0 representing non-compliance and 100 representing complete compliance. Coping self-efficacy changes came from a unit-weighted factor score $(\alpha=0.73)$ that consisted of the changes (within-subject regression coefficients) for three subscales from the Arthritis Self-Efficacy scale - coping self-efficacy with pain, functioning, and other symptoms over time ${ }^{37}$ To estimate the change process, we first computed slope estimates over time for the Arthritis Self-Efficacy subscales using a linear mixed-effects model, saved the random coefficients for each participant, and partialled out baseline effects (ie, the random coefficient for the intercept) to only change by participant. Thus, the first mediator (compliance) was a treatment process variable, while the second mediator (coping self-efficacy) represented a proximal treatment change mechanism variable.

\section{Physical functioning}

We combined four Short Form-36 Health Survey subscales to create a composite measure of physical functioning change - a composite computed via the same unit-weighted factor score used previously. ${ }^{38}$ The subscales consisted of the total scale, physical functioning, bodily pain, and physical health. Since research participants' physical functioning changed due to treatment, we estimated change scores from linear mixed-effects models (ie, the random coefficients with partialled out intercepts) just as we estimated the changes in coping self-efficacy. These change scores reflect the change in physical functioning and do not include any variance attributable to baseline functioning prior to treatment. Thus, all physical functioning change should be attributable to the traits (promotion or prevention for this model), treatment compliance, or treatment target (coping self-efficacy changes) and not on pre-treatment physical functioning.

\section{Data analysis}

We tested a two-mediator (compliance and changes in coping self-efficacy) path model where promotion and prevention effects on changes in physical functioning were mediated by treatment compliance and changes in coping self-efficacy (ie, the proximal treatment target). Our rationale for the ordering of the mediation pathway is as follows. First, the dispositional factors of promotion and prevention are viewed as traits rather than states and accordingly occur prior to any treatment. Thus, these factors served as our antecedents $(\mathrm{X})$ in our mediation model. Second, compliance (M1) is a treatmentrelevant process variable measured throughout the 3-month treatment period. Treatment, therefore, logically follows our antecedents $(\mathrm{X})$ and precedes changes in coping self-efficacy and physical functioning. Finally, since the treatment focused on changing coping self-efficacy we assumed that changes in that proximal outcome would precede changes in physical functioning. We assessed the model using the structural equation model (SEM) package in $\mathrm{R}$ (version 2.15.2) ${ }^{39,40}$ - a program that uses the ML algorithm to produce parameter estimates and standard errors. Our choice of SEM to analyze the mediational model was due to its flexibility in allowing us to test both mediational pathways simultaneously. Model fit evaluation was based upon the standard chi-square (nonsignificant), comparative fit index (CFI; $>0.9$ ), and root mean square error of approximation (RMSEA; <0.05) standards. 
All bivariate relationships in the path model were subjected to regression analysis and diagnostics to rule out regression artifacts, outlier influence, non-linear relationships, or spurious effects. In addition to this SEM model, we conducted a dummy-coded bivariate general linear model to examine the simple impact of a person's promotion and prevention profile on changes in overall functioning.

\section{Results}

A total of 182 participants ( $72 \%$ of the baseline sample) completed all follow-up measures throughout the 2-year follow-up period. Missing data were handled with multiple imputation using predictive mean-matching in the R statistical package "mice", ${ }^{41}$ and indicated a negligible amount of missing information (mean $\gamma<0.00001$ ). Dropout can be seen in the consort diagram (Figure 1). The analysis sample was all 254 participants from baseline. Participants were on average $52.6(\mathrm{SD}=7.1$ ) years old, $77.0 \%$ women, $91.7 \%$ white, and with an average BMI of $27.7 \mathrm{~kg} / \mathrm{m}^{2}(\mathrm{SD}=4.2)$. As reported in the original intervention study, some of these demographic characteristics influenced functioning, ${ }^{30}$ however, none of these factors significantly moderated the meditational path model presented, and thus are not included to preserve parsimony of the results.

The two-mediator model met all fit criteria. Specifically, the CFI (0.99) met our criterion of 0.90 , the chi-square was non-significant $\left(\chi^{2}[1]=0.14, p=0.70\right)$, and the RMSEA was within our criterion of 0.05 (RMSEA $<0.01$; 95\% CI: $0,0.12)$. We inspected model modification indices and none improved our model nor would they be theoretically justifiable. Thus, we chose to report the model specified a priori and to ignore empirically driven modifications. In total, the prediction model accounted for $16 \%$ of the variance in coping self-efficacy change and $27 \%$ of the variance in physical functioning change.

Table 2 lists the coefficients and appropriate statistics for our resultant model. Each parameter represents a path in Figure 2 with standardized coefficients and their appropriate error terms, probability values, and requisite $\mathrm{p}_{\text {rep }}$ values. Specifically, promotion had a positive, direct effect $(\beta=0.22$, $z=3.21, p<0.05)$ - and no significant indirect effect - on changes in physical functioning. Higher promotion scores predicted greater positive changes in physical functioning over the 2-year study. By contrast, prevention had a negative indirect effect - and no significant direct effect - on physical functioning. Higher prevention scores predicted lower or even negative changes in compliance $(\beta=-0.13, z=-2.13, p<0.05)$ and coping self-efficacy $(\beta=-0.26, z=-3.65, p<0.05)$. Coping
Table 2 SEM parameters and statistics

\begin{tabular}{lllllll}
\hline Path & Beta & $\beta$ & $\mathbf{S E}_{\mathrm{b}}$ & $\mathbf{z}$ & $\mathbf{p}$-value & $\mathbf{P}_{\text {rep }}$ \\
\hline Prevention $\rightarrow$ compliance & -0.13 & -0.07 & 0.03 & -2.13 & 0.03 & 0.93 \\
Prevention $\rightarrow$ self-efficacy & -0.23 & -0.38 & 0.10 & -3.65 & 0.00 & 1.00 \\
Promotion $\rightarrow$ self-efficacy & 0.11 & 0.16 & 0.10 & 1.73 & 0.08 & 0.91 \\
Prevention $\rightarrow$ functioning & 0.02 & 0.12 & 0.11 & 1.06 & 0.29 & 0.80 \\
Promotion $\rightarrow$ functioning & 0.23 & 0.32 & 0.10 & 3.21 & 0.00 & 0.99 \\
Compliance $\rightarrow$ self-efficacy & 0.22 & 0.66 & 0.17 & 3.80 & 0.00 & 0.99 \\
Compliance $\rightarrow$ functioning & 0.02 & 0.10 & 0.19 & 0.53 & 0.60 & 0.63 \\
Self-efficacy $\rightarrow$ functioning & 0.42 & 0.43 & 0.07 & 6.41 & 0.00 & 1.00 \\
Prevention $\leftrightarrow$ promotion & -0.56 & -0.20 & 0.03 & -7.86 & 0.00 & 1.00 \\
\hline
\end{tabular}

Notes: " $\rightarrow$ " refers to directional association with temporal precedence. " $\leftrightarrow$ " refers to correlational association within the same time point.

Abbreviation: SEM, structural equation model.

self-efficacy changes had a positive relationship with changes in physical functioning $(\beta=0.40, z=6.41, p<0.05)$. Thus, higher prevention scores were predictive of lower treatment compliance and lower or even negative physical functioning changes but those effects were fully mediated by changes in coping self-efficacy (see Figure 2). Promotion and prevention measures showed a significant, negative correlation $(\beta=-0.57, z=-7.86, p<0.05)$.

With respect to the process-related variables, compliance directly and positively predicted changes in coping self-efficacy $(\beta=0.22, z=-3.80, p<0.05)$. With regard to the path model with promotion orientation as the primary predictor, four of the nine specified paths were significantly different from zero, with a fifth being marginally significantly different from zero. Figure 2 shows the complete model along with the significant standardized paths and their respective coefficients. The significant paths all had relatively high probability estimates for replication ( $p_{\text {rep }}>0.60$ for all). Bivariate regression diagnostic tests revealed no significant data points to skew the results.

In order to get a general idea of how the four potential promotion/prevention profiles predicted the main treatment outcome, physical functioning, we created binary data by

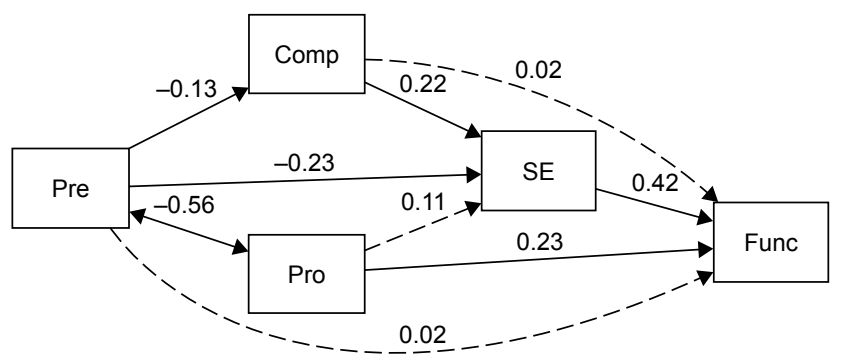

Figure 2 Path model with standardized parameter estimates of significant paths. Notes: Solid lines represent significant paths. Dashed lines represent nonsignificant paths.

Abbreviations: Pre, prevention orientation; Pro, promotion orientation; Comp, changes in compliance; SE, changes in self-efficacy; Func, changes in functioning. 


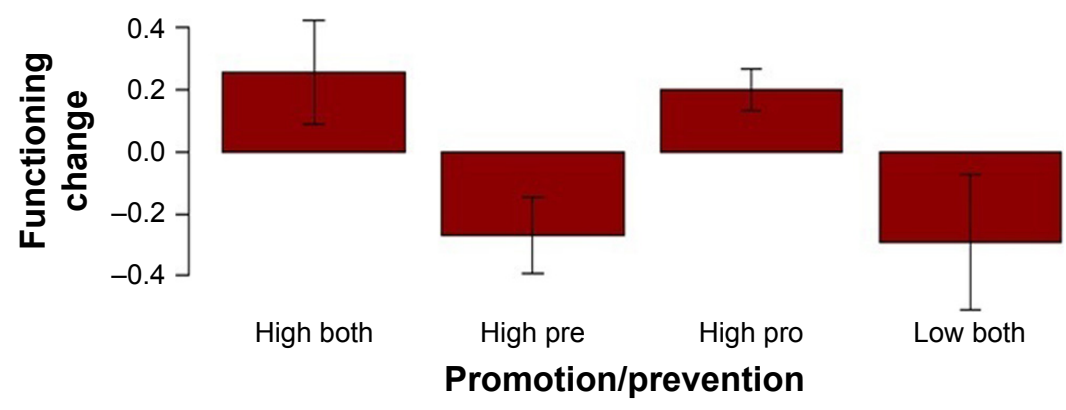

Figure 3 Percentage of participants with improved functioning by median-split promotion/prevention classification profiles. Note: Bars below 0.0 indicate more participants in this group decreased in functioning than increased in functioning. Abbreviations: Pre, prevention; Pro, promotion; Both, prevention and promotion.

splitting - via median - all participants' promotion and prevention score to categorize them as high on both, low on both, or high on one and low on another. We observed large differences in the percentage of participants who increased in functioning by promotion/prevention profile: more participants high in promotion and low in prevention improved in functioning $(59 \%$ improved, $\mathrm{N}=103$ ), slightly more participants high in both promotion and prevention improved in functioning ( $53 \%$ improved, $\mathrm{N}=38$ ), while more participants actually decreased in functioning (44\% improved, $\mathrm{N}=38$ ) if they were low in both promotion and prevention; considerably more participants decreased in functioning ( $35 \%$ improved, $\mathrm{N}=71$ ) if they were low in promotion and high in prevention (Figure 3 ).

Participants low in both promotion and prevention showed a marginal decrease in functioning $(\beta=-0.29$, $p=0.055$ ). Compared to participants low in both promotion and prevention, participants high in promotion orientation actually showed a positive change in functioning. This was true for participants solely high in promotion $(\beta=0.49$, $p=0.007$ ) and participant high in both promotion and prevention ( $\beta=0.55, p=0.013$ ). By contrast, participants high in prevention not only did not improve but also decreased in functioning similar to participants low in both prevention and promotion $(\beta=0.02, p=0.907)$. There were no significant effects for these between-group analyses when examining compliance and changes in coping self-efficacy.

\section{Discussion}

We provided simple and striking evidence for the utility of classifying individuals based upon promotion and prevention orientation. Furthermore, we provided potential mechanistic evidence for two distinct paths from promotion and prevention orientations to treatment outcomes. A promotion orientation predicts increased functioning directly; promotion-oriented individuals improved more in functioning, and this improvement could not be accounted for by greater compliance or coping self-efficacy over the course of the intervention. So what is the mechanism by which a trait promotion orientation directly leads to reporting of increased functioning? It is our belief that people with a promotion orientation report better functioning because they are not focused on functioning. Instead, they are focused on achieving any number of other future goals that are personally meaningful, ${ }^{42}$ which is the end-game of any treatment. ${ }^{43}$ Focusing on these end goals may in fact serve as a resilience factor to negative experiences along the way. ${ }^{44} \mathrm{~A}$ deeper look at our findings supports this conclusion.

\section{Promotion and prevention orientations and treatment outcomes}

We have a clear view of how the treatment outcome itself differed by a participant's standing on promotion and prevention. Very simply, profiles high in promotion were likely to show improvements in functioning, whereas profiles high in prevention were less likely to show improvements, and even showed decreases in functioning (as seen in Figure 3). Most strikingly, having low promotion scores was consistently related to a greater chance of decreases in functioning across these profiles. These functioning differences provide a clear message: having promotion-oriented goal is necessary to see improvements in functioning, regardless of a person's prevention-oriented goal. As previously mentioned, this may reflect the need for individuals to have some future-oriented goal to strive toward in order to benefit maximally from this particular treatment. This invokes the notion that promotion and prevention orientations might also be proxy variables for other related individual difference factors, including a person's temporal focus. ${ }^{45}$ Supplementing these results, our SEM results provide nuanced detail about promotion and prevention as individual predictors in the treatment process. 


\section{Promotion orientation in the treatment process}

While promising as an individual predictor, promotion orientation failed to predict either of the treatment process variables. We had hypothesized that a promotion orientation would predict treatment compliance - a simple, intuitive assumption given that compliance ought to be an indicator of promotion-oriented behavior. Yet, the act of showing up to a treatment session provides an imperfect behavioral indicator of treatment engagement (mindful engagement might be better).$^{46}$ Furthermore, promotion orientation did not predict changes in self-efficacy - the treatment change process targeted in the treatment trial. We expected a promotion orientation to be relevant to dynamic goal-oriented processes. While the exact mechanism that accounts for a relationship between promotion orientation and coping self-efficacy cannot be determined from our available data, it is possible that promotion-oriented individuals already maintained reasonable levels of coping self-efficacy and did not require any changes to initiate functional outcome changes. Our hypothesis of promotion orientation predicting compliance may not be borne out with these analyses; however, compliance did predict changes in coping self-efficacy and changes in coping self-efficacy predicted changes in functioning. Thus, there is still a clear path between treatment processes and outcomes, which appear to capture the sequelae of prevention-focused individuals more so than promotion-focused individuals.

The influence of both compliance and promotion orientation on physical functioning was mediated by coping self-efficacy; neither showed a direct effect. These different mediational processes may be indicative of the cognitive (promotion orientation) and behavioral (treatment compliance) components of most psychosocial interventions for degenerative diseases. In some studies, the cognitive component (eg, positive coping strategies) $)^{47}$ significantly predicted outcomes, while in others, the behavioral component (eg, treatment compliance) ${ }^{48}$ significantly predicted outcomes. Both appear relevant to the change process but they exert a differential impact on the eventual outcomes.

\section{Prevention orientation in the treatment process}

Although related to the treatment process, it appears that a prevention orientation provides little value in directly predicting treatment response - at least for osteoarthritis sufferers. Theories largely based upon the similar framework of prevention and avoidance (eg, Terror Management Theory $)^{49}$ might predict certain negative behaviors well but may not predict positive change processes such as overcoming health problems, struggling with life stressors, or attaining difficult goals. Recent research suggests that temperament interacts with the treatment type. ${ }^{11,50}$ Individuals presented with congruent treatment messages (ie, promotion oriented with positive messages and prevention oriented with negative messages) responded best to treatment. All three treatment arms in the original Knee study were positively framed such that participants who were more promotion-oriented might respond to the treatment. These findings might fit well with the aptitude by treatment interactions $;{ }^{14}$ however, further evidence needs to clearly support this treatment matching effect.

\section{Limitations}

Taken together, these findings suggest that treatment response may be predicated on promotion orientations and not on prevention orientations. These findings are consistent with previous work but there are some limitations that need elaboration. Both promotion and prevention orientations were observed and not selected - raising the possibility that the effects might be overestimated for one (promotion) and underestimated for the other (prevention). These biases might arise from a lack of variance due to self-selection into the study or variability in orientation related to osteoarthritis. In defense of the latter problem, we selected only individuals who were predominantly inactive, and so it is unlikely that these people were more likely to be promotion-oriented individuals if promotion orientation were predictive of physical activity. We expect the contrary and hold that these results might actually be a conservative estimate of the effects of each temperament.

Another potential limitation is that osteoarthritis might offer a unique situation that fails to generalize well to other psychological or medical conditions. We acknowledge that limitation but hold that arthritis, in general, serves as an excellent proving ground for psychosocial theories related to stress and well-being. Arthritis impacts both physical and cognitive aspects of daily life and provides equal opportunity for individual differences of behavioral temperament and cognitive vulnerabilities to impact outcomes. These degenerative diseases provide us with an opportunity to observe how individual differences may affect both cognitive functioning and physical functioning over time. Nevertheless, our findings may not generalize to other less stressful or single-faceted diseases. 


\section{Strengths and future directions}

Strengths of the current research include the focus on a real-world sample of adults within a treatment study and an examination of psychological and physical change over time. The simultaneous analysis of multiple dispositions and mediators in the same model underscores the positive psychological tenant of the independence of positive and negative. Our findings show promise for studying how personality and social psychological constructs and theories can help clinical psychology differentiate treatment responders from non-responders. Future research focusing on individual differences may unravel other potentially useful aptitude by treatment interactions that seem hard to identify. ${ }^{11,14}$ In addition, future research may shed more light on a reciprocal relationship between individuals and treatment, whereby treatment also affects psychological factors of an individual (eg, a prevention-oriented patient acquiring a promotionoriented state in treatment). While there is substantial work to be done, we feel the promise from these findings should stimulate more interest in this line of inquiry.

\section{Disclosure}

Dan V Blalock was supported by grant number TPH 21-000 from the Department of Veterans Affairs Office of Academic Affiliations. Patrick E McKnight, was supported by the National Institute of Arthritis, Musculoskeletal, and Skin Diseases; R01-AR-047595. Todd B Kashdan was supported by the Center for the Advancement of Well-Being at George Mason University. The authors report no other conflicts of interest in this work.

\section{References}

1. Keyes CL. Mental illness and/or mental health? Investigating axioms of the complete state model of health. J Consult Clin Psychol. 2005; 73(3):539-548.

2. Namburi P, Beyeler A, Yorozu S, et al. A circuit mechanism for differentiating positive and negative associations. Nature. 2015;520(7549): 675-678.

3. Carver CS, Sutton SK, Scheier MF. Action, emotion, and personality: emerging conceptual integration. Pers Soc Psychol Bull. 2000;26(6): 741-751.

4. Wollburg E, Braukhaus C. Goal setting in psychotherapy: the relevance of approach and avoidance goals for treatment outcome. Psychother Res. 2010;20(4):488-494.

5. Strauman TJ. Self-regulation and psychopathology: toward an integrative translational research paradigm. Ann Rev Clin Psychol. 2017;13: 497-523.

6. Marszalek J, Price LL, Harvey WF, Driban JB, Wang C. Outcome expectations and osteoarthritis: association of perceived benefits of exercise with self-efficacy and depression. Arthritis Care Res (Hoboken). 2017; 69(4):491-498.

7. Waters S, Strauman T, Keefe F, Linton C, Caldwell D. The relationship of self-discrepancies to pain and depression in osteoarthritis patients. J Pain. 2005;6(3):S59.
8. Selten EM, Geenen R, Schers HJ, et al. Treatment beliefs underlying intended treatment choices in knee and hip osteoarthritis. Int J Behav Med. 2018;25(2):198-206.

9. Higgins ET. Beyond pleasure and pain. Am Psychol. 1997;52(12): $1280-1300$.

10. Higgins ET. Making a good decision: value from fit. Am Psychol. 2000; 55(11):1217-1230

11. Covey J. The role of dispositional factors in moderating message framing effects. Health Psychol. 2014;33(1):52-65.

12. Broderick JE, Schneider S, Junghaenel DU, Schwartz JE, Stone AA. Validity and reliability of patient-reported outcomes measurement information system instruments in osteoarthritis. Arthritis Care Res (Hoboken). 2013;65(10):1625-1633.

13. Burisch M. Approaches to personality inventory construction: a comparison of merits. Am Psychol. 1984;39(3):214-227.

14. Smith B, Sechrest L. Treatment of aptitude X treatment interactions. J Consult Clin Psychol. 1991;59(2):233-244.

15. Higgins ET. Value from regulatory fit. Curr Dir Psychol Sci. 2005; 14(4):209-213.

16. Haidt J, Rodin J. Control and efficacy as interdisciplinary bridges. Rev Gen Psychol. 1999;3(4):317-337.

17. Stretcher V, DeVellis M, Becker M, Rosenstock I. Self-efficacy and the health belief model. Health Educ Q. 1986;13:73-92.

18. Rothman AJ, Salovey P. Shaping perceptions to motivate healthy behavior: the role of message framing. Psychol Bull. 1997;121(1):3-19.

19. O'Keefe DJ, Jensen JD. The relative persuasiveness of gain-framed loss-framed messages for encouraging disease prevention behaviors: A meta-analytic review. J Health Commun. 2007;12(7):623-644.

20. Wang J, Lee AY. The role of regulatory focus in preference construction. J Mark Res. 2006:28-38.

21. Keller PA, Lehmann DR. Designing effective health communications: a meta-analysis. J Public Policy Mark. 2008;27(2):117-130.

22. Latimer AE, Rivers SE, Rench TA, et al. A field experiment testing the utility of regulatory fit messages for promoting physical activity. J Exp Soc Psychol. 2008;44(3):826-832.

23. Fuglestad PT, Rothman AJ, Jeffery RW. Getting there and hanging on: the effect of regulatory focus on performance in smoking and weight loss interventions. Health Psychol. 2008;27(3S):S260-S270.

24. Lee AY, Aaker JL. Bringing the frame into focus: the influence of regulatory fit on processing fluency and persuasion. J Pers Soc Psychol. 2004;86(2):205-218.

25. Silverwood V, Blagojevic-Bucknall M, Jinks C, Jordan JL, Protheroe J, Jordan KP. Current evidence on risk factors for knee osteoarthritis in older adults: a systematic review and meta-analysis. Osteoarthr Cartil. 2015;23(4):507-515.

26. Fransen M, McConnell S. Exercise for osteoarthritis of the knee. Cochrane Database Syst Rev. 2008;(4):CD004376.

27. Fransen M, McConnell S, Harmer AR, Van der Esch M, Simic M, Bennell KL. Exercise for osteoarthritis of the knee: a Cochrane systematic review. Br J Sports Med. 2015;49(24):1554-1557.

28. Devos-Comby L, Cronan T, Roesch SC. Do exercise and self-management interventions benefit patients with osteoarthritis of the knee? A metaanalytic review. J Rheumatol. 2006;33(4):744-756.

29. Towheed TE, Maxwell L, Judd MG, Catton M, Hochberg MC, Wells G. Acetaminophen for osteoarthritis. Cochrane Database Sys Rev. 2006; (1):CD004257.

30. McKnight PE, Kasle S, Going S, et al. A comparison of strengthtraining, self-management, and the combination for early osteoarthritis of the knee. Arthritis Care Res (Hoboken). 2010;62(1):45-53.

31. Kellgren JH, Lawrence JS. Radiological assessment of rheumatoid arthritis. Ann Rheum Dis. 1957;16(4):485-493.

32. Higgins ET, Friedman RS, Harlow RE, Idson LC, Ayduk ON, Taylor A. Achievement orientations from subjective histories of success: promotion pride versus prevention pride. Eur J Soc Psychol. 2001;31(1):3-23.

33. Watson D, Clark LA, Tellegen A. Development and validation of brief measures of positive and negative affect: the PANAS scales. J Pers Soc Psychol. 1988;54(6):1063-1070. 
34. Costa PT Jr, McCrae RR. Stability and change in personality assessment: the revised NEO personality inventory in the year 2000. J Pers Assess. 1997;68(1):86-94.

35. Radloff LS. The CES-D scale: a self-report depression scale for research in the general population. Appl Psychol Meas. 1977;1:385-401.

36. Grice JW, Harris RJ. A comparison of regression and loading weights for the computation of factor scores. Multivariate Behav Res. 1998;33(2): 221-247.

37. Lorig K, Holman H. Arthritis self-efficacy scales measure self-efficacy. Arthritis Care Res. 1998;11(3):155-157.

38. Ware JE Jr, Sherbourne CD. The MOS 36-item short-form health survey (SF-36). I. conceptual framework and item selection. Med Care. 1992;30(6):473-483.

39. Fox J, Kramer A, Friendly M. sem: Structural equation models. (R package version 0.9-20). 2010. Available from: https://cran.r-project. org/web/packages/sem/sem.pdf. Accessed April 24, 2017.

40. R Development Core Team. R: a language and environment for statistical computing. Vienna: R Foundation for Statistical Computing; 2010 ISBN 3-900051-07-0.

41. van Buuren S, Groothuis-Oudshoorn K. Mice: multivariate imputation by chained equations in R. J Stat Softw. 2011;45(3):1-67.

42. McKnight PE, Kashdan TB. Purpose in life as a system that creates and sustains health and well-being: an integrative, testable theory. Rev Gen Psychol. 2009;13(3):242-251.

43. Hayes SC, Villatte M, Levin M, Hildebrandt M. Open, aware, and active: contextual approaches as an emerging trend in the behavioral and cognitive therapies. Annu Rev Clin Psychol. 2011;7:141-168.
44. Blalock DV, Young KC, Kleiman EM. Stability amidst turmoil: Grit buffers the effects of negative life events on suicidal ideation. Psychiatry Res. 2015;228(3):781-784.

45. Zimbardo PG, Boyd JN. Putting time in perspective: a valid, reliable individual-differences metric. J Pers Soc Psychol. 1999;77(6) 1271-1288.

46. Brown KW, Ryan RM, Creswell JD. Mindfulness: theoretical foundations and evidence for its salutary effects. Psychol Inq. 2007;18(4): 211-237.

47. Burns JW, Kubilus A, Bruehl S, Harden RN, Lofland K. Do changes in cognitive factors influence outcome following multidisciplinary treatment for chronic pain? A cross-lagged panel analysis. J Consult Clin Psychol. 2003;71(1):81-91.

48. van den Ende CH, Hazes JM, le Cessie S, et al. Comparison of high and low intensity training in well controlled rheumatoid arthritis. Results of a randomised clinical trial. Ann Rheum Dis. 1996;55(11):798-805.

49. Greenberg J, Pyszczynski T, Solomon S. The causes and consequences of a need for self-esteem: a terror management theory. In: Baumeister R, editor. Public Self and Private Self. New York: Springer-Verlag; 1986: 189-212.

50. Gerend MA, Shepherd MA. When different message frames motivate different routes to the same health outcome. Ann Behav Med. 2015 50(2):319-329.
Patient Preference and Adherence

\section{Publish your work in this journal}

Patient Preference and Adherence is an international, peer-reviewed, open access journal that focuses on the growing importance of patient preference and adherence throughout the therapeutic continuum. Patient satisfaction, acceptability, quality of life, compliance, persistence and their role in developing new therapeutic modalities and compounds to optimize

\section{Dovepress}

clinical outcomes for existing disease states are major areas of interest for the journal. This journal has been accepted for indexing on PubMed Central. The manuscript management system is completely online and includes a very quick and fair peer-review system, which is all easy to use. Visit http://www. dovepress.com/testimonials.php to read real quotes from published authors. 